\title{
Revision total hip arthroplasty using the Zweymuller femoral stem
}

\author{
Matthew E. Oetgen · Michael H. Huo • \\ Kristaps J. Keggi
}

Received: 11 January 2008/ Accepted: 6 March 2008/Published online: 10 May 2008

(C) Springer-Verlag 2008

\begin{abstract}
Background A variety of femoral stem designs have been reported to be successful in revision total hip arthroplasty without consensus as to optimal design. We evaluated the clinical and radiographic outcomes in a consecutive series of femoral revisions using a wedge-shape, tapered-stem design at medium and long-term follow-up.

Materials and methods We performed a retrospective review of clinical and radiographic outcomes of twentyeight consecutive femoral revisions arthroplasties, which were done using the Zweymuller femoral stem.

Results The mean follow-up was 7.4 years (range 2-15 years). No stem re-revision was necessary. All stems were judged to be stable by radiographic criteria at the most recent follow-up. The final mean Harris hip score was 90 . There was no difference in Harris hip scores, implant stability, or radiological appearance (distal cortical
\end{abstract}

The authors have no financial relationship with any product mentioned in this manuscript. We have full control of all primary data and agree to allow the journal to review the data if requested.

M. E. Oetgen $(\varangle) \cdot$ K. J. Keggi

Department of Orthopedics and Rehabilitation,

Yale University School of Medicine,

P.O. Box 208071, New Haven, CT 06520, USA

e-mail: matthew.oetgen@yale.edu

\section{K. J. Keggi}

Keggi Orthopedic Foundation, Turnpike Office Park,

1579 Straits Turnpike, Middlebury, CT 06762, USA

\section{H. Huo}

Department of Orthopedic Surgery,

University of Texas Southwestern Medical Center,

1801 Inwood Road, Dallas, TX 75390-8883, USA hypertrophy or proximal stress shielding) of the implants between medium-term (mean 5.7 years) and long-term (mean 12.4 years) follow-up.

Conclusions We found the Zweymuller femoral stem design to be durable for revision hip arthroplasty when there is an intact metaphyseal-diaphyseal junction for adequate press-fit stability at surgery.

Keywords Total hip arthroplasty · Revision · Femoral component

\section{Introduction}

Total hip arthroplasty (THA) is one of the most successful orthopedic reconstructive operations. Improvements in design, biomaterial, and surgical techniques have led to increased durability of THAs. Despite these improvements and innovations, failures do occur and revision surgeries are necessary. Revision rates for aseptic failure have been reported between $1.5 \%$ and nearly $20 \%$ in mid- to longterm follow-up [1]. Many stem designs and surgical techniques have been utilized in revision of the femoral stem. These include: cement fixation [2], cementless proximallycoated stems [3], extensively-coated stems [4], and modular stems $[5,6]$. Regardless of the stem design, the principle pre-requisites for femoral revision are: maximizing fit, immediate press-fit stability, control of axial and rotational stability, and optimal bone-remodeling in the long-term. Variable success rates have been reported with each design and technique.

The purpose of this study was to evaluate the clinical and radiographic outcome in a consecutive series of femoral revisions done by a single senior surgeon using a unique stem design at mid-term follow-up. 


\section{Materials and methods}

The senior surgeon began using the Zweymuller (AlloClassic) (Zimmer, Warsaw, IN) stem design in January 1988. This stem design was extended to revision THAs in March, 1988. The senior surgeon performed 210 femoral revisions between March 1988 and November 1992 using a variety of stems. 166 of these revisions were performed using cementless fixation; of these, 28 were done using the Zweymuller stem design. This particular stem design was chosen for femoral revision in cases with an intact femoral meta-diaphyseal junction based upon preoperative templating and intraoperative assessment of femoral bone stock, and in cases in which reconstruction of leg length and femoral offset could be preformed with this stem design. The current study was conducted to review all the revisions done using the Zweymuller stem, which represented a consecutive, unselected series using this particular stem design.

\section{Demographics}

There were 18 men and 10 women. The mean age was 66 years (range $48-83$ years). The mean BMI was $29 \mathrm{~kg}$ / $\mathrm{m}^{2}$ (range $24.6-37.4 \mathrm{~kg} / \mathrm{m}^{2}$ ). On average, the patients had 1.8 previous hip surgeries prior to the latest revision. Femoral revision was indicated for: failed cemented stems (18 hips), failed cementless stems (9 hips), and failed bipolar hemiarthroplasty (1 hip). The mean interval between the index hip surgery and the most recent revision was 10.2 years.

Femoral bone deficiency was graded by a single observer using the AAOS classification system using preoperative AP and lateral radiographs [7]. Eight (29\%) of the patients had no significant femoral bone deficiency. Sixteen patients (57\%) had only small cavitary and segmental bone deficiency near the calcar or the greater trochanter, whereas, four patients (14\%) did have segmental deficiency of $>2.5 \mathrm{~cm}$.

\section{Stem design}

The Zweymuller stem design is unique in its biomaterial, geometry, and surface texture. The biomaterial is a titanium-alloy composed of titanium with $6 \%$ aluminum and $4 \%$ niobium (Ti-6Al-4N) used to fabricate the femoral stems. It has a rectangular cross-section. It is a wedgeshape tapered design with single-plane taper in the lateralmedial dimension while the anterior-posterior dimension remains constant. Inventory included 12 stem sizes ranging from 110 to $168 \mathrm{~mm}$ in length. The stem surface is not porous. The texture is slightly roughened with a mean interspace size ranging from 4 to 6 microns. It offers a
Morse taper neck design that can accept modular femoral head components with either Co-Cr-alloy or ceramic surface.

\section{Surgical technique}

All revisions were done using the modified anterior approach developed by the senior surgeon [8]. This versatile approach can be used for either minimal-incision technique, or more extensile exposure. It has been utilized in over 7,000 THAs. We have reported the clinical efficacy and outcome of revision THAs $[8,9]$.

This muscle splitting approach utilizes the interval between the sartorius medially, and the tensor fasciae lata laterally. The abductor mechanism is left undisturbed. Capsular release is done to expose the upper femur and the acetabulum. Acetabular revision was done in 23 patients. Femoral stem and cement removal were done by standard techniques, without the need for femoral shaft osteotomy. Femoral canal preparation was performed using rasps alone. The stem size was determined by fit within the canal. In selected cases, if lengthening was required from preoperative planning, a larger stem size was selected to achieve seating at a more proximal position within the canal to restore soft tissue tension, offset, and leg length.

All patients received identical prophylaxis protocol for antibiotics and thromboembolism. All patients were allowed to begin full-weight activity immediately following surgery.

\section{Follow-up}

All patients were entered into a prospective database. They were followed routinely at 4-6 weeks after surgery, at 6 months, 1 year, and annually thereafter. Clinical evaluation was done using the Harris hip scale [10]. All radiographs were taken using identical protocol with nondigital technique by the same technicians over the study period. Radiographic evaluation was done following the criteria previously published by our group [11].

Statistical analysis was done using Student $t$ test to assess the significance of continuous variables, and a Pearson chi-square test to assess the significance of categorical variables. Significance was defined as $P$ value less than 0.05. All patients did give consent for inclusion in this study.

\section{Results}

Four patients (4 hips) did not return for a minimum 2-year follow-up, thus were excluded. The final analysis was therefore done in the remaining 24 hips (Table 1, 2). No 
Table 1 Patient data-medium-term follow-up (16 patients)

\begin{tabular}{|c|c|c|c|c|c|c|c|c|c|}
\hline Patient & $\begin{array}{l}\text { Bone } \\
\text { deficiency }\end{array}$ & $\begin{array}{l}\text { HSS } \\
\text { pre }\end{array}$ & $\begin{array}{l}\text { HSS } \\
\text { post }\end{array}$ & $\begin{array}{l}\text { Follow-up } \\
\text { (years) }\end{array}$ & Complications & $\begin{array}{l}\text { Stress } \\
\text { shielding }\end{array}$ & Hypertrophy & $\begin{array}{l}\text { Anterior } \\
\text { thigh pain }\end{array}$ & $\begin{array}{l}\text { Pain meds } \\
\text { at } F / U\end{array}$ \\
\hline 1 & None & 17 & 93 & 6.1 & None & None & None & No & No \\
\hline 2 & Large segmental & 66 & 100 & 5.5 & None & None & IT & No & No \\
\hline 3 & Small cavitary & 43 & 87 & 5.7 & None & None & None & No & No \\
\hline 4 & Small cavitary & 35 & 89 & 3.3 & Stem settling & None & None & No & No \\
\hline 5 & None & 40 & 68 & 3.6 & None & LT & Medial tip & Yes & Yes \\
\hline 6 & None & 44 & 97 & 8.6 & None & None & Medial tip & No & No \\
\hline 7 & Large segmental & 32 & 91 & 5.6 & None & IT & Medial tip & No & Yes \\
\hline 8 & Small cavitary & 40 & 100 & 7.5 & None & None & None & No & No \\
\hline 9 & Small segmental & 18 & 86 & 6.6 & None & GT & LT & No & No \\
\hline 10 & None & 43 & 72 & 2 & None & None & Medial and lateral tip & Yes & Yes \\
\hline 11 & Small cavitary & 58 & 100 & 4.6 & None & None & None & No & No \\
\hline 12 & Small segmental & 44 & 84 & 2 & Calcar fracture & None & Medial and lateral tip & No & No \\
\hline 13 & None & 40 & 59 & 8.1 & None & None & None & Yes & Yes \\
\hline 14 & Small cavitary & 24 & 75 & 9.4 & None & None & None & No & No \\
\hline 15 & None & 38 & 86 & 6.5 & Periprosthetic fracture & None & None & No & No \\
\hline 16 & Small segmental & 42 & 97 & 6.4 & None & None & None & No & No \\
\hline
\end{tabular}

Table 2 Patient data-long-term follow-up (8 patients)

\begin{tabular}{|c|c|c|c|c|c|c|c|c|c|}
\hline Patient & $\begin{array}{l}\text { Bone } \\
\text { deficiency }\end{array}$ & $\begin{array}{l}\text { HSS } \\
\text { pre }\end{array}$ & $\begin{array}{l}\text { HSS } \\
\text { post }\end{array}$ & $\begin{array}{l}\text { Follow-up } \\
\text { (years) }\end{array}$ & Complications & $\begin{array}{l}\text { Stress } \\
\text { shielding }\end{array}$ & Hypertrophy & $\begin{array}{l}\text { Anterior } \\
\text { thigh pain }\end{array}$ & $\begin{array}{l}\text { Pain meds } \\
\text { at } F / U\end{array}$ \\
\hline 1 & Small cavitary & 28 & 97 & 13.5 & Greater trochanter fracture & None & None & No & No \\
\hline 2 & Small cavitary & 41 & 100 & 15 & None & None & None & No & No \\
\hline 3 & Large segmental & 54 & 91 & 13.7 & $\begin{array}{l}\text { - Acetabular revision } \\
\text { - Calcar fracture }\end{array}$ & IT & Medial and lateral tip & No & No \\
\hline 4 & None & 52 & 100 & 13.3 & None & None & None & No & No \\
\hline 5 & None & 45 & 95 & 11.1 & None & IT & Medial tip & No & No \\
\hline 6 & Small cavitary & 59 & 90 & 11.7 & None & LT & None & No & No \\
\hline 7 & Large segmental & 53 & 100 & 10.4 & None & None & Mid stem & No & No \\
\hline 8 & Small cavitary & 55 & 92 & 10.5 & None & None & None & No & No \\
\hline
\end{tabular}

stem was re-revised for any reason. The mean follow-up was 7.4 years (range $2-15$ years). The mean Harris hip score improved from a preoperative mean of 42-90 points at final follow-up. There was no significant difference in the mean Harris hip scores at the 2-year (82 [95\% CI:7194]), 5-year (87 [95\% CI:81-92]), and 10-year (96 [95\% CI:88-104]) intervals $(P=0.10)$. Eight patients died during the follow-up interval. In this group, all hips were reported by family members to be doing well at the time of the patients death. The remaining 16 patients were all functioning well at last follow-up. The mean Harris score for the living patients was 90 (range 59-100). Three patients experienced occasional anterior thigh pain related to activities; four patients (including the three with thigh pain) took occasional analgesics for pain related to their THAs.
Complications included: one greater trochanter, and two calcar fractures during surgery. Circlage wires were used for the calcar fractures, while no treatment was required for the trochanter fracture (Fig. 1). All patients did well without any residual problems related to their fractures. One additional patient sustained a femoral shaft fracture from trauma 2 years after surgery that was successfully treated with open reduction and internal fixation without stem revision. There was no infection, dislocation, or clinical thromboembolism in the series. Brooker [12] II heterotopic ossification was seen in one hip. The patient's functional status was not altered. One isolated acetabular revision was done 14 years after the index revision and the stem was found to be stable at that time.

Radiographic evaluation demonstrated stable stem fixation in all hips (100\%) (Fig. 2). One stem did subside 
Fig. 1 a AP pre-revision radiograph. b Post operative radiograph of revision right THA using Zweymuller stem. Notice the fractured greater trochanter. c, d 8-year follow-up radiographs. e ,f 14-year followup radiographs showing solid ingrowth, minimal proximal bony resorption, and no evidence of loosening
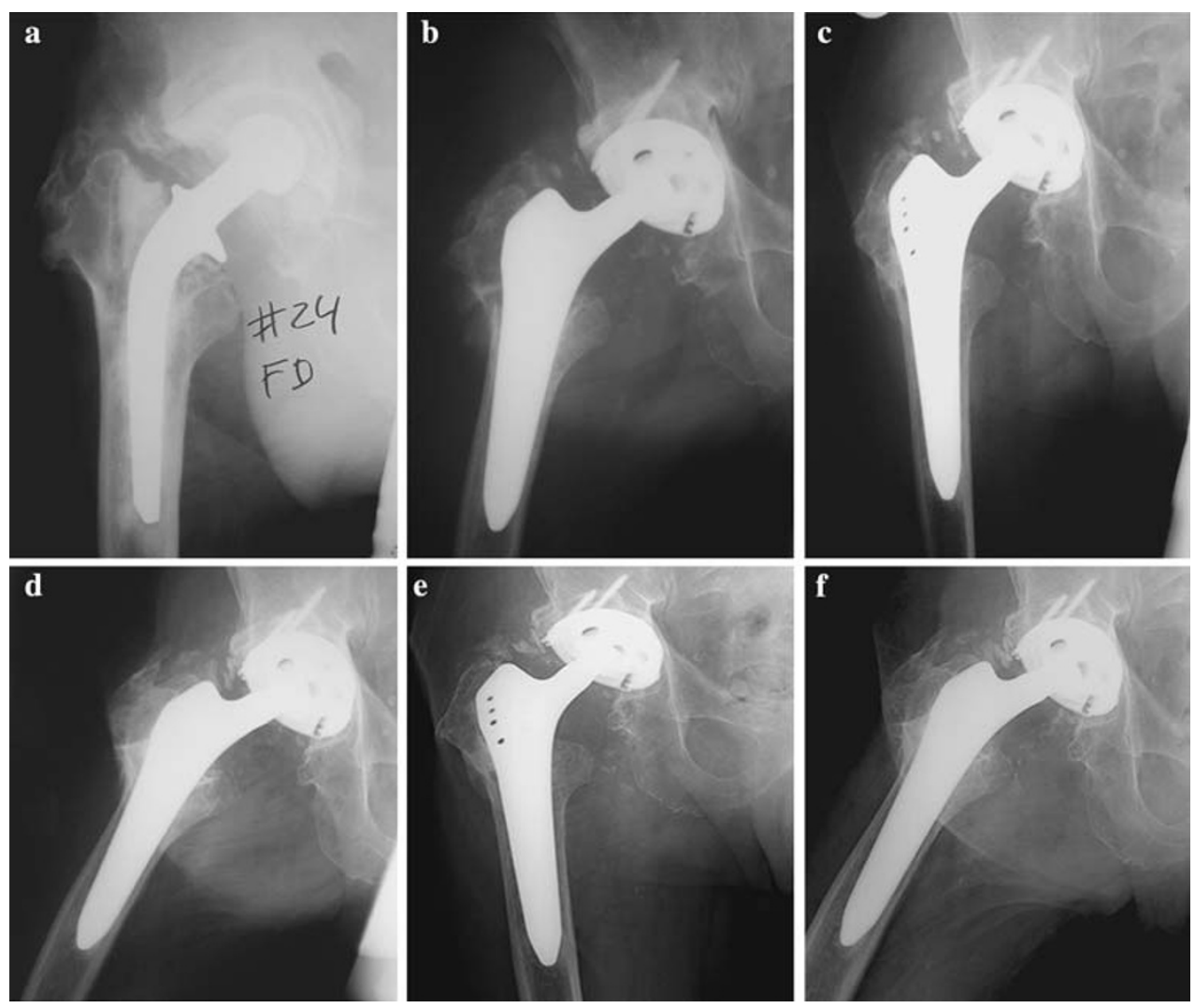

$>5 \mathrm{~mm}$ within the first month after surgery. This patient did not have a calcar fracture. The stem stabilized, and has since gone on to show radiographic evidence of bony ingrowth with good clinical outcome. This case illustrated the utility of a tapered stem geometry in that subsidence could result in greater axial and rotational stability leading to durable stem fixation. Distal cortical hypertrophy (Gruen zone 4) was observed in ten patients (42\%). Proximal stress-related bone remodeling (stress shielding) was observed in six patients $(25 \%)$. No quantitative measurement of stress shielding was done. No femoral or pelvis osteolysis was observed.

We elected to analyze the data further by breaking the patients into two groups based upon mean follow-up time. One group (long-term) included 8 hips with a mean followup of 12.4 years (range 10-15 years). The other group (medium-term) included 16 hips with a mean follow-up of 5.7 years (range $2-9$ years). We were especially interested in determining if there was a difference between the 2 groups with regard to clinical outcome, and bone remodeling changes. Tables 3 and 4 show the results for these 2 groups. There was no difference in the Harris hip scores between the groups, pre- or post-op $(P=0.091$ and $P=0.056$, respectively). There was no difference in fixation stability, distal cortical hypertrophy, or proximal stress shielding between the groups.

\section{Discussion}

Recent data from the National Hospital Discharge Survey showed both the total number and rate of primary and revision total hip arthroplasty (THA) procedures are increasing [13]. In addition to the increase in number of procedures, analysis of this data by age groups showed this trend to continue across both young and elderly patients. The annual revision burden remained relatively constant at $17.5 \%$ per year over the study period. It is expected that the total number of revision THAs will continue to rise with increasing primary THAs being done, especially in younger and more active patients. Moreover, increases in revision THAs are at least in part due to longer patient life expectancy and more active life style. Improvements in implant design and surgical techniques for revision THAs have led to much better clinical outcome over the past three decades. There are however limitations and suboptimal results of the existing techniques. Efforts in developing and evaluating the efficacy and durability of newer and alternative designs and techniques must be continued.

Cementless fixation has evolved to be the predominant technique in femoral revisions. The commonly used stems include: (1) extensively-coated cylindrical design; (2) proximally-coated cylindrical or anatomically-shaped designs; and (3) modular designs with a variety of 


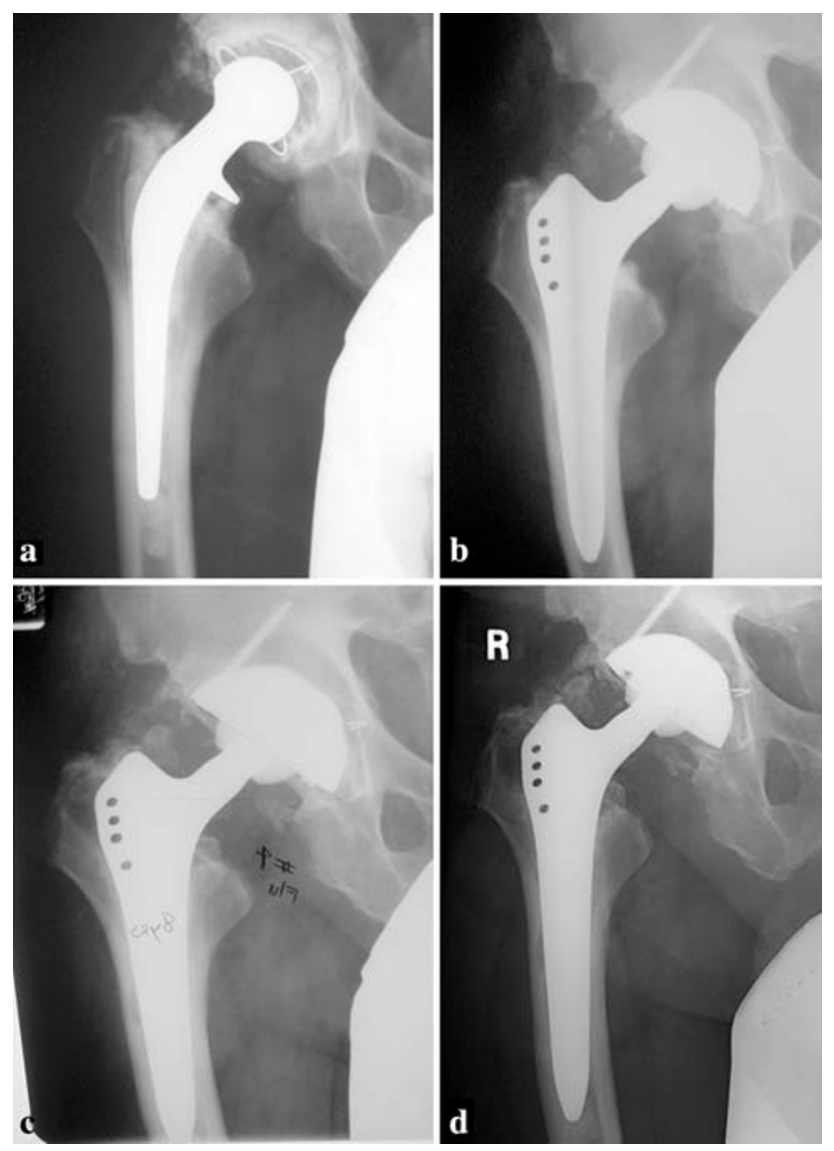

Fig. 2 a AP pre-revision radiograph. b Post operative radiograph of revision right THA using Zweymuller stem. c 8-year follow-up radiographs. d 15-year follow-up radiograph, with some evidence of stress shielding of the greater trochanter

Table 3 Clinical data

\begin{tabular}{llll}
\hline Follow-up group & $\begin{array}{l}\text { Medium term } \\
(n=16)\end{array}$ & $\begin{array}{l}\text { Long term } \\
(n=8)\end{array}$ & $P$ Value \\
\hline Harris hip score (pre) & $39(32-45)$ & $48(39-57)$ & 0.091 \\
Harris hip score (post) & $87(81-92)$ & $96(88-103)$ & 0.056 \\
Anterior thigh pain & 3 & 0 & 0.19 \\
$\begin{array}{c}\text { Patients needing pre-op } \\
\text { pain medication }\end{array}$ & 11 & 4 & 0.37 \\
$\begin{array}{c}\text { Patients needing pain } \\
\text { medication at last F/U }\end{array}$ & 4 & 0 & 0.12 \\
\hline
\end{tabular}

Harris hip scores reported as mean $(95 \% \mathrm{CI})$

combinations of proximal and/or distal fit. Most techniques require rigid fit in the femoral diaphysis. Paprosky et al. [4] reported their experience in 170 extensively porous-coated stems. These THAs were followed for a mean of 13 years. Overall stem survival at 15 years was $95 \%$. Radiographic evaluation demonstrated bony-stable stem fixation in $82 \%$, and fibrous-stable fixation in $14 \%$ of the hips. Six femoral stems were re-revised due to loosening. Similar clinical
Table 4 Radiographic data

\begin{tabular}{llll}
\hline Follow-up group & $\begin{array}{l}\text { Medium term } \\
(n=16)\end{array}$ & $\begin{array}{l}\text { Long term } \\
(n=8)\end{array}$ & $P$ value \\
\hline Bony ingrowth & $100 \%(16 / 16)$ & $100 \%(8 / 8)$ & \\
Subsidence & $6 \%(1 / 16)$ & $0 \%(0 / 8)$ & 0.47 \\
Distal cortical hypertrophy & $44 \%(7 / 16)$ & $38 \%(3 / 8)$ & 0.77 \\
Proximal stress shielding & $19 \%(3 / 16)$ & $38 \%(3 / 8)$ & 0.31 \\
\hline
\end{tabular}

efficacy and fixation durability have also been reported with modular stem designs. Christie et al. [5] reported on 129 revisions using the S-ROM stem (DePuy a Johnson \& Johnson Company. Warsaw, IN). The mean follow-up was 6.2 years. Overall stem survival at 10 years was $98 \%$. Radiographic evaluation demonstrated bony-stable fixation of the stems in $92 \%$. Clinical efficacy was reflected in an increase of the Harris hip score from 47 (preoperative) to 87 (final follow-up). One femoral component underwent re-revision for mechanical loosening. Kwong et al. [6] reported on the short-term results of using the Link modular tapered stem (Waldemar Link GmbH \& Co, Hamburg, Germany) in 143 revision THAs. The overall stem survival rate was $97 \%$ at 5 years. Moreover, the mean Harris hip score at final follow-up was 92. A total of 4 femoral stems underwent re-revision, one for infection, one due to surgical error, and 2 due to mechanical component failure.

The Zweymuller femoral stem is designed with a unique geometry, which provides several advantages when used in revision THAs. The rectangular cross-section and wedgeshaped tapered geometry allows for four-point fixation along the four corners within the femoral canal. The fixation is not dependent upon filling of the diaphysis such as in the case of either cylindrical extensively-coated, or modular straight/tapered stem designs. It does require an intact metaphysic-diaphysis junction. Extensive femoral bone deficiencies such as Paprosky IIIa or IIIb [4] may be challenging, especially without longer length of this particular stem design to gain fixation in the upper diaphysis.

There is the additional advantage of no required diaphyseal reaming in femoral canal preparation. This may avoid inadvertent distal perforations or fractures. Avoidance of reaming also in theory preserves greater femoral canal blood flow to maximize bone healing potential around the stem. We found bony-stable fixation in $100 \%$ of our stems. Fixation did not deteriorate between the medium-term (5+ years), and the longer-term (12 years) follow-up groups. Finally, we did not observe any significant qualitative adverse bone remodeling as we compared sequential radiographs for each patient. Stress shielding has been widely reported with the use of extensively-coated stems with stable distal fixation $[14,15,16]$.

Care must be taken with preparation and insertion of this stem. Its wedge-shape geometry has the potential to result 
in proximal femur fractures by a log splitting mechanism. We did not find this to be a limitation in the clinical outcome. There were two (7\%) calcar fractures, which required circlage wire fixation in the 28 THAs. This compares well with fracture incidence of using extensively porous-coated and modular stem designs in revision THA. Some have reported fracture rate of $9-30 \%[4,5,17]$.

We believe the Zweymuller femoral stem provides reliable stable fixation in femoral revision surgery. We have had no case of fixation failure up to 15 years in this relatively small series. This technique is especially useful in those cases where there is no extensive segmental bone deficiency in the proximal femur.

Acknowledgments The authors would like to thank Laurine E. Zatorski, RN for her assistance in this project.

Conflict of interest statement The authors declare that they have no conflict of interest related to the publication of this manuscript.

\section{References}

1. Callaghan JJ, Rosenberg AG, Rubash HE (1998) The adult hip, 1st edn. Lippincott-Raven, Philadelphia

2. Haydon CM, Mehin R, Burnett S et al. (2004) Revision total hip arthroplasty with use of a cemented femoral component. Results at a mean of 10 years. J Bone Joint Surg Am 86:1179-1185

3. Emerson RH Jr, Head WCHiggins LL (2003) Clinical and radiographic analysis of the Mallory-Head femoral component in revision total hip arthroplasty. A minimum 8.8-year and average 11-year follow-up study. J Bone Joint Surg Am 85:1921-1926

4. Paprosky WG, Greidanus NV, Antoniou J (1999) Minimum 10year results of extensively porous-coated stems in revision hip arthroplasty. Clin Orthop 369:230-242

5. Christie MJ, DeBoer DK, Tingstad EM et al (2000) Clinical experience with a modular noncemented femoral component in revision total hip arthroplasty: 4-7-year results. J Arthroplasty 15:840-848

6. Kwong LM, Miller AJ, Lubinus P (2003) A modular distal fixation option for proximal bone loss in revision total hip arthroplasty: a 2-6-year follow-up study. J Arthroplasty 18:94-97

7. D'Antonio J, McCarthy JC, Bargar WL et al. (1993) Classification of femoral abnormalities in total hip arthroplasty. Clin Orthop 296:133-139

8. Kennon R, Keggi J, Zatorski LE et al. (2004) Anterior approach for total hip arthroplasty: beyond the minimally invasive technique. J Bone Joint Surg Am 86(Suppl 2):91-97

9. Kennon RE, Keggi JM, Wetmore RS et al. (2003) Total hip arthroplasty through a minimally invasive anterior surgical approach. J Bone Joint Surg Am 85(Suppl 4):39-48

10. Harris WH (1969) Traumatic arthritis of the hip after dislocation and acetabular fractures: treatment by mold arthroplasty. An endresult study using a new method of result evaluation. J Bone Joint Surg Am 51:737-755

11. Huo MH, Martin RP, Zatorski LE et al (1995) Total hip arthroplasty using the Zweymuller stem implanted without cement. A prospective study of consecutive patients with minimum 3-year follow-up period. J Arthroplasty 10:793-799

12. Brooker AF, Bowerman JW, Robinson RA et al. (1973) Ectopic ossification following total hip replacement. Incidence and a method of classification. J Bone Joint Surg Am 55:16291632

13. Kurtz S, Mowat F, Ong K et al (2005) Prevalence of primary and revision total hip and knee arthroplasty in the United States from 1990 through 2002. J Bone Joint Surg Am 87:1487-1497

14. Engh CA, McGovern TF, Bobyn JD et al. (1992) A quantitative evaluation of periprosthetic bone-remodeling after cementless total hip arthroplasty. J Bone Joint Surg Am 74:1009-1020

15. Keisu KS, Mathiesen EB, Lindgren JU (2001) The uncemented fully textured Lord hip prosthesis: a 10-15-year follow-up study. Clin Orthop 382:133-142

16. Engh CA Jr, Young AM, Engh CA Sr et al. (2003) Clinical consequences of stress shielding after porous-coated total hip arthroplasty. Clin Orthop 417:157-163

17. Meek RMD, Garbuz DS, Masri BA et al. (2004) Intraoperative fracture of the femur in revision total hip arthroplasty with a diaphyseal fitting stem. J Bone Joint Surg Am 86:480-485 